\title{
Preoperative Anxiety in Patients Undergoing Outpatient Cancer Surgery
}

J. Majumdar, E. Vertosick, B. Cohen, M. Levine, M. Assel \& M. Barton-Burke

\section{Introduction}

The diagnosis of cancer may evoke anxiety in patients related to death, pain, disfigurement, disability, and disruption of relationships. ${ }^{[1]}$ In addition, the possibility of surgery creates an additional source of anxiety for many patients. A number of outpatient cancer surgeries are almost entirely performed on an outpatient basis including lumpectomies of the breast (97\%), plastic reconstruction procedures (79\%), and gynecology procedures $(72 \%)^{[2]}$.

Our primary research aim was to determine the prevalence of preoperative anxiety in patients undergoing outpatient cancer surgery. Our secondary aim was to examine the association between preoperative anxiety and negative intraoperative and postoperative outcomes in patients undergoing outpatient cancer surgery, including increased anesthesia requirements, postoperative nausea and vomiting (PONV), unplanned overnight stay, length of stay, transfer, surgical complications, and postoperative visits to urgent care centers (UCC).

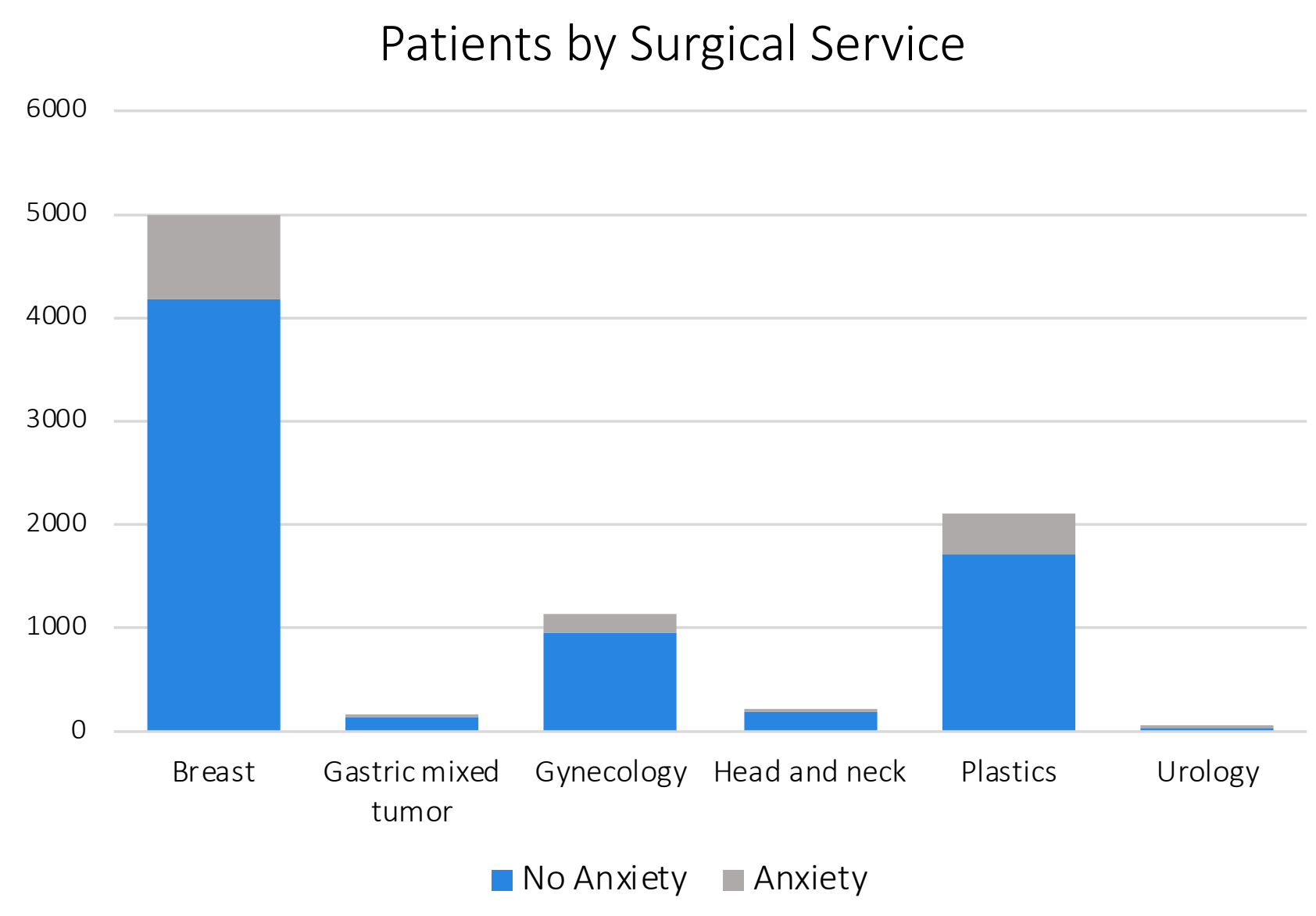

\section{Methods}

We conducted a retrospective cohort study to investigate the prevalence of preoperative anxiety and its association with postoperative outcomes in patients undergoing outpatient cancer surgery. Our retrospective cohort included 10,048 outpatient procedures performed on 8683 patients at a large comprehensive cancer center between January 1, 2016, and April 30, 2018.

Preoperative anxiety was identified if the patient had an anxiety care plan initiated by nursing staff in the preoperative setting and recorded in the EMR, indicating that the patient's anxiety required additional nursing intervention.

\section{Results}

$16.7 \%$ of the patients experienced preoperative anxiety according to nursing assessment.

Our multivariable analysis demonstrated that patients with preoperative anxiety had higher rates of adverse outcomes including postoperative nausea and vomiting (PONV) $(P=$ 0.029), unplanned overnight admission $(P=0.021)$, and urgent care visits within 30 days $(P=0.002)$.

\section{Conclusions}

In our cohort of patients undergoing outpatient cancer surgery, nearly one in five patients was identified by nursing assessments to be experiencing notable levels of preoperative anxiety. Our findings highlight the importance of assessing and recording preoperative anxiety in patients undergoing outpatient cancer surgery so that future studies can attempt to elucidate the causal pathway between preoperative anxiety and postoperative adverse events.

\section{Postoperative Events}

No Preoperative Anxiety

Postoperative Length of Stay $(n=8373)$

Conversion from MAC to general anesthesia $(n=4786), n(\%)$

Any use of postoperative PONV medication $(n=8665), n(\%)$

Same day resurgery $(n=8665), n(\%)$

Transfer from Outpatient Surgery Center $(n=8665), n(\%)$

Overnight stay $(n=8626), n(\%)$

Grade 3 or higher complication within 30 days $(n=8665), n(\%)$

UCC visit within 30 days $(n=8665), n(\%)$

Readmission within 30 days $(n=8665), n(\%)$

\section{References}

1. Deng G, Cassileth BR. Integrative oncology: Complementary therapies for pain, anxiety, and mood disturbance. CA Cancer J Clin 2005;55:109-16.

\begin{tabular}{|c|c|c|}
\hline $2.9 \mathrm{~h}$ & $3.0 \mathrm{~h}$ & 0.13 \\
\hline 505 (13) & $83(11)$ & 0.13 \\
\hline 715 (10) & $181(13)$ & 0.029 \\
\hline $27(0.4)$ & $12(0.8)$ & 0.022 \\
\hline $17(0.2)$ & $4(0.3)$ & 0.7 \\
\hline $184(2.6)$ & $54(3.8)$ & 0.021 \\
\hline $11(0.2)$ & $8(0.6)$ & 0.006 \\
\hline $183(2.5)$ & $58(4.0)$ & 0.002 \\
\hline $104(1.4)$ & $34(2.4)$ & 0.008 \\
\hline
\end{tabular}

\section{Acknowledgements}

This research was supported in part through the $\mathrm{NIH} / \mathrm{NCl}$ Cancer Center Support Grant P30 CA008748, the Kimmel Center for Prostate and Urologic Cancer, and the Geri \& ME Nursing Fund Grant.

2. Steiner C, Karaca Z, Moore B, Imshaug M, Pickens G. Surgeries in Hospital-Based Ambulatory Surgery and Hospital Inpatient Settings, 2014. HCUP Statistical Brief \#223; 2017. Available from: http://www.hcup-us.ahrq.gov/reports/statbriefs/sb223Ambulatory-Inpatient-Surgeries-2014.pdf. 